ARID International Journal of Social Sciences and Humanities (AIJSSH) VOL.4, NO.7, January 2022

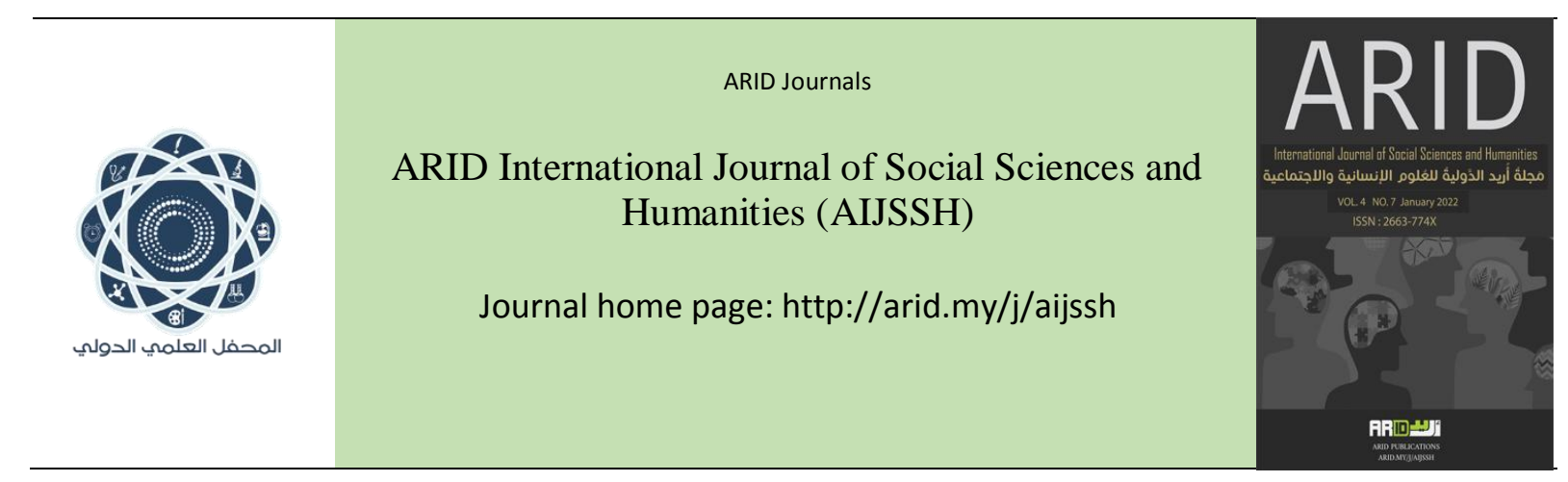

$$
\begin{aligned}
& \text { مَجلةُ أُريد الدَّوليةُ للعُلومِ الإنسانية والإجتماعية } \\
& \text { العدد السابع، البملد الرابع، كانون الثاني } 2022 \text { م }
\end{aligned}
$$

\title{
Sustainable development and ethics question vision finality
}

\author{
Okba DJENANE
}

Mohamed KHIDER University of Biskra-Algeria-Faculty of Human and Social Sciences

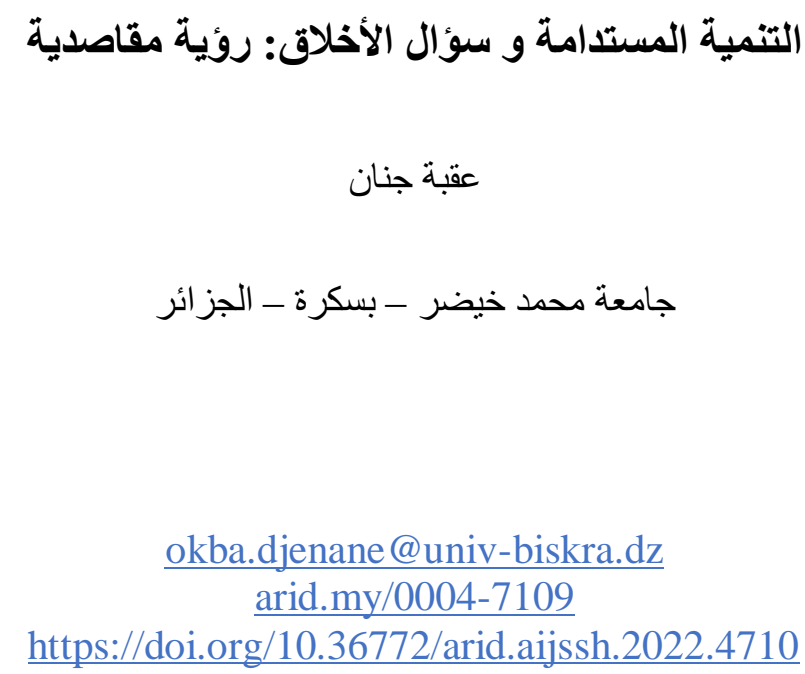




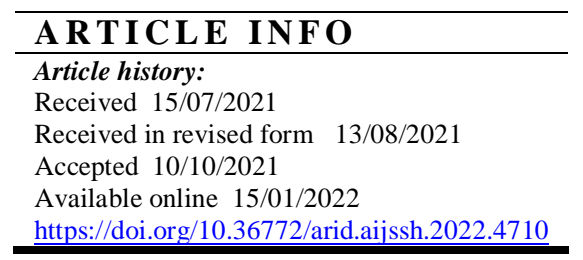

\begin{abstract}
The problem of sustainable development emerged in today's environmental crisis is mainly caused by the Western modernist model of consumption. The later fails to preserve the environment and keeps its promises for sustaining it. Their failure questions, in myriad of ways, the ethical dimensions of human attachment to their environment. The present paper aims at investigating the contribution of people's ethical commitment in protecting the environment in creating the fair balance between the demands of present generations and the future generations. It attempts also to approach the question of sustainable development from applied philosophy and Islamic ethics perspectives. The study uses the analytical and descriptive method to deal with the problematic relationship between ethics and sustainable development. It concludes that the lack of the environmental ethics hinders establishing a sustainable development for our environment.
\end{abstract}

Key words: Sustainable development, environmental ethics,Islamic ethic, Jurisprudence,Applied Philosophy. 


\section{الملخص}

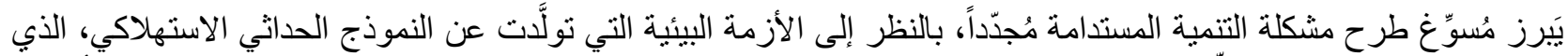

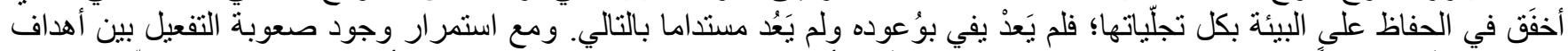

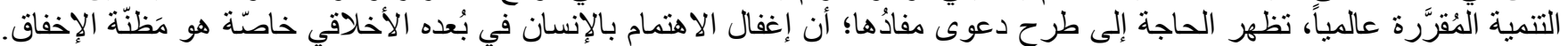

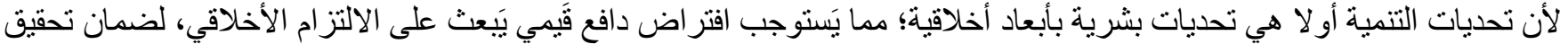

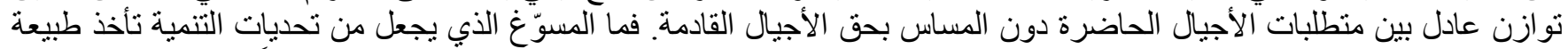

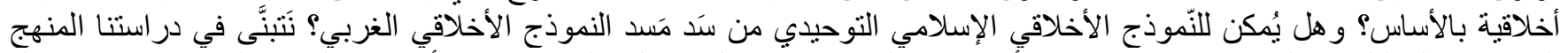

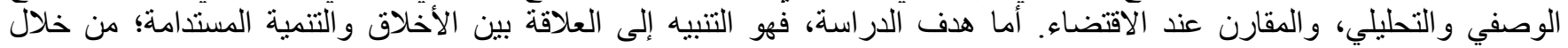

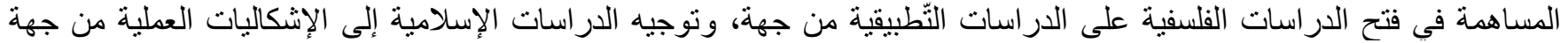

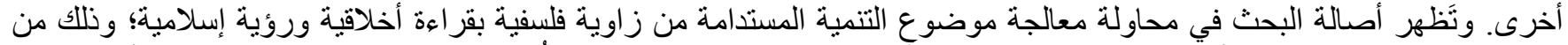

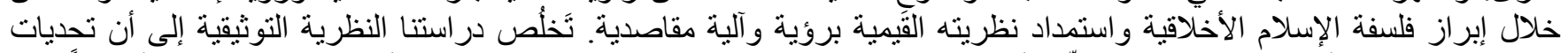

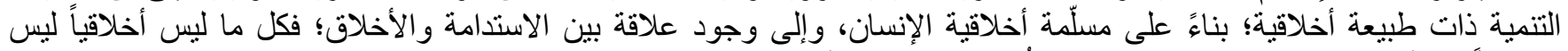

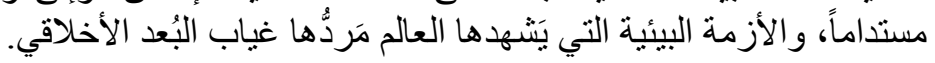

الكلمات المفتاحية: التنمية المستدامة، الأخلاق البيئية، الأخلاق الإسلامية، الاجتهاد المقاصدي، الفلسفة التطبيقية. 


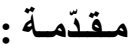

يُعتبر مفهوم " التتمية المستدامة " من المفاهيم التي أفرزنها العولمة وتداعياتها؛ فهو مفهوم في طور الإنجاز ، ما انفكّ يأخذ صوراً مختلفة وأبعاداً متعددة، بعد أن أظهر تمَلملا في خُطط التنمية المقترحة؛ لإخر اج الدول الفقيرة من و اقعها المُزري، وأبان عن عدم كفاية الفكر الاقتصادي و عدم صو ابيّة التناول المادي للمفهوم، مما أدّى إلى ضرورة البحث عن فكر جديد وتبنّي نهج آخر يتجاوز مفهوم التنمية الاقتصادية إلى مفهوم التتمية الثاملة، التي تأخذ بعين الاعتبار الأبعاد غير المادية؛ أي مجموع التغيرات العميقة والمعقّة التي تمسّ الأبعاد الاجتماعية و السياسية والثقافية المُمتدّة عبر الزمن[1]. زيادة على ذلك، يُلحّ محمول "الاستدامة" على موضوع "التتمية" أن تأخذ، إلى جانب الأبعاد الأخرى- بُعداً أخلاقيا؛ تَتمّلّه الأنشطة الإنسانية في الحفاظ على الموروث البشري والطبيعي فلا تطاله يَّ العبث. فوجُود مقصد الاستدامة أو طلب الديمومة في التنمية دليل على ضرورة استدعاء عامل قيمي أو بُحد أخلافي؛ من شأنه أن يَرفع اللّبس أو التناقض Oxymoron في المفهوم الذي يُقِّم نفسه

$$
\text { على أنه مفهوم أخلاقي جو هرياً][2]. }
$$

و عليه، كانت ضرورة إدر اج الأبعاد الأخلاقية والاعتبار ات القيمية في تحقيق الثروات وتوزيعها توزيعا عادلا، مع زيادة الاهتمام بالبيئة الذي انبنَى عليه مفهوم الاستدامة أصلا. لذلك حاول خبر اء أخلاقيات البيئة ومنظمات الأعمال الوصول إلى وُجهات نظر منقاربة ومتقاطعة، ثمّ توسيع الالنزامات الأخلاقية وتوظيفها في الأبعاد الاقتصادية والبيئية، لتحقيق حياة معتدلة على كل الصُّعد الإنسانية و الحيو انية والنباتية و الثيئانيةة][3]. ولو أن هذه المرحلة، التي وُصفت بالثّاملة، أعطت قدراً من الاهتمام الأخلاقي للمشكلة التتموية؛ في اهتمامها بالأبعاد الإنسانية وتقدير ها للممارسات الجالبة للمصلحة والدّافعة للمضرّة. إلا أنها بَقبت رهينة الأبعاد المادية، الأمر الذي يدفعنا من جهة؛ إلى تسجيل عجز أو عدم كفاية على الأقل في النموذج التنموي الغربي؛ الذي كان استجابة طبيعية لآليات النموذج الحداثي الاستهلاكي بكل تمظهر اته. ومن جهة أخرى افتر اض دافع قَيمي "عُلوي و مُهيمن" يضمن الحفاظ على التنمية دون فساد أو ضرر. فهل يُككن للنّموذج الأخلافي الإسلامي الذي يَنبني أساساً على مفهوم "المصلحة والصلاح" من أن يكون حافظا للتنمية وجودا و عدماً، فيسَد بالتالي مَسد النموذج الأخلاقي الغربي في حل تحدّي التنمية ؟ كما يبرُز سؤال الاعتبار ات الأخلاقية في نشأة المفهوم، إلى جانب البحث عن المسوّغ الذي يجعل من تحديات التنمية ذات طبيعة أخلاقية بالدرجة الأولى. تكمن أهمية دراستتا في محاولة معالجة موضوع التنمية المستدامة من زاوية فلسفية بقراءة أخلاقية ورؤية إسلامية خاصة؛ مرتكزين في ذلك على منظومة الإسلام القيمية والأخلاقية ممثّلة في علم المقاصد. الذي يمثل إجابة جديدة ومتجددة؛ بالنظر إلى 
موضو عاته المبنية على مسايرة مشكلات العصر و أسئلته المستجدة و الحائرة ! ومنه، تكون المساهمة في استئناف وتوسيع مجال الاجتهاد في فلسفة الدين؛ الذي هو اجتهاد في التفكير الفلسفي أساساً. أما هدف الدراسة فيتمثل في؛ استحضار وَعي قيمي وأخلاقي وفق المرجعية الإسلامية، وذلك قصد الوصول إلى تشكيل تصوّر عن مفهوم التنمية، يتماشَى مع فلسفة الإسلام الكونية؛ القائمة على فكرة الاستخلاف و مارة الأرض. زيادة على كثف عجز الجهد الحداثي في بلورة أخلاق كافية وقادرة لسد المثلب القيمي، الذي كان نتيجة إلغاء السند الاعتقادي للقيم والأخلاق في بعدها الذّيني وصيغتها الإسلامية. لمعالجة إثكالية البحث وتحقيقا لأهدافه، أرتأينا تناول الموضوع من خلال المطالب التالية:

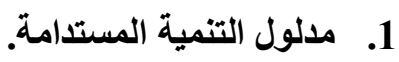
2. معضلة نموذج التنمية العلماني الأخلاقية. 3. الأخلاق البيئية. 4. التمية بلحاظ المقاصد. أ. أ. المقاصد و الاستدامة. ب. أخلاقية المقاصد. ت. مدلول التتمية برؤية مقاصدية. ثم نختم الدراسة بعرض أهم النتائج و التوصيات المتعلقة بالموضوع. 1 بَرز مفهوم "التنمية المستدامة" لأول مرة في مؤتمر ستوكهولم حول البيئة الإنسانية سنة 1972، فكان بمثابة خطوة نَحو الاهتمام العالمي بالبيئة؛ إذ تعرَّض المؤتمر لأول مرة للقضايا البيئية وعلاقاتها بالفقر وغياب التتمية في العالم. فبَعد أن انتقَّ الدّول التي تتجاهل البيئة أثناء عملية التخطيط للتنمية، دعاها لاتخاذ التدابير اللازمة لحمايتها. وبصدُور قرار المنظور البيئي عن جمعية الأمم المتحدة عام 1987، الذي كان هدفه تحقيق التنمية البيئية المستدامة، تمّ لأول مرة تقديم تعريف التنمية المستدامة[4]. لكن مفهوم التّمية لم يَستقر على تصوّر واحد؛ خاصّة في موضوع العمارة والعمران، وهذا نظراً لصعوبة تحديد الأولويات المطلوب تحقيقها في المشاريع المختلفة، مما أدّى إلى تجدّده المستمر وتولّدّ كمّ كبير من المفاهيم المتباينة ؛ الأمر الذي جعل المفهوم لا يظهر على صورة واحدة. لكنه يجتهد في الحفاظ على فكرة مركزية مفادُها "تحسين مستوى حياة الأجيال الحاضرة مع الحفاظ على حق الأجيال القادمة في العيش"؛ أي 》ا افتراض بأن القرار ات الحالية يجب ألا تُضِفِ من إمكانية الحفاظ أو تحسين مستوى الحياة بالمستقبل 
من خلال إدارة جيدة للنّظم الاقتصادية إلى تحقيق ربحية الموارد وصيانة الأصول الثانية.؛ [5]؛ و على نفس الأساس تعتبرها اللجنة العالمية للتنمية المستدامة على أنها : 》ا تلبية احتياجات الحاضر دون أن تؤدي إلى تدمير قدرة الأجيال المقبلة على تلبية احتياجاتها الخاصِّة.ب[2].

وعرّفها تقرير برونتلاند Brundtland سنة 1987على أنها : 》 عملية للتغيير يتناغم فيها استغلال الموارد وتوجهات الاستثمار و وناحي التنمية التكنولوجية وتغيير المؤسسات ويُعزّز كلا من إمكانات الحاضر و المستقبل للوفاء باحتياجات الإنسان وتطلعاته.؛ [2]؛ أي الاجتهاد في تغيير نمط الاستهلاك الحالي، واستبداله بنمط استهلاكي يضمن تحقيق إنتاجية مستدامة، ودون هذا المنهج لا يمكن بحال تحقيق الاستدامة في التنمية؛ فزيادة على حماية البيئة من جهة و الحفاظ على مستقبل الأجيال القادمة، دمَج التقرير بين البُعد الاقتصادي والاجتماعي و البيئي معاً. وبعد تطوّر مفهوم التنمية Development، لم يَعد المصطلح بعني مجرّد النّمو Growth الاقتصادي و التراكم المادي، بل أخذ في سباق العولمة معنئ آخر؛ مُؤدّاه الجمع و الفصل بين التحوّلات الاقتصادية والمالية والتحوّلات البسيكولوجية والاجتماعية والسياسية و المؤسساتية][3]. وكذا نوازن وتكافؤ الجانب المادي والمعنوي البيئي والاجتماعي لحاضر الإنسان ومستقبله. وفي إطار إبراز البُعد المعنوي يُشتير François Perreux إلى أن التنمية هي :» عملية تنسيق التغيرات الذهنية والاجتماعية لشعب ما والتي تؤدّي إلى رفع مستوى الإنتاج الحقيقي الإجمالي بشكل تر اكمي ودائم.ب[6]. فالتنمية المستدامة بهذا المعنى، ترمي إلى محاولة مُلاعمة الضّروريات الأساسية لجميع الناس وتحقيق التوازن بين التفاعلات والتغيرات للعلاقات المتبادلة؛ والتي تَخصنّ الإنسان بكل إمكانياته وثقافته وحضارته و تفاعله مع البيئة استغلالا أو تعديلاً أو تتمية][7]. إن التنمية المستدامة هي تنمية إنسانية أولاً، غايتها تأهيل الإنسان روحيا وتعليميا وثقافيا وصحياً، مع تلبية حاجاته الضرورية، زيادة على تحقيق قيم المواطنة كالحرية والمساواة و العدالة الاجتماعية. و هي تتمية مرهونة باحترام البيئة وأخذ جو انبها بعين الاعتبار. لذلك يؤكّ Amartya Sen صاحب نظرية القدرات و الوظائف، على أنها صيرورة متكاملة لترقية الحريات الأساسية في شكل متر ابط ؛ تأخذ على إثر ها التنمية مقاربة أخلاقية، تتمثل في المسؤولية تجاه العالم الفقير من جهة والبيئة وما يتعرّض له كوكب الأرض من جهة أخرى[8]. وتتميّز التتمية المستدامة عن التنمية التقليدية بالاستمرارية والتواصل؛ لأنها معيار نجاح العملية التنموية. زيادة على تنظيم استخدام المو اد الطبيعية المتجددة والقابلة للنفاد؛ بما يَضمن حق الأجيال القادمة فيها. أما المعيار الضابط للتنمية المستدامة فهو المحافظة على سلامة الحياة الطبيعية و إنتاج ثروات متجدّدة. وأخير ا، تَقصد التنمية المستدامة إلى تحقيق التكامل بين الاقتصاد و المجتمع و البيئة. و إذا كانت مؤشرات التنمية التقليدية، تقيس التخيُر الذي يطر أ على جانب ما؛ بالنّظر إليه على أنه مؤشر مستقِل، فإن القياس في التنمية المستدامة يأخذ بعين الاعتبار تداخل و تَعلّق المؤشرات بعضها ببعض؛ بحيث ينعكس أب تغير على الجوانب الأخرى[9]. وييقى التكامل 
بين أبعاد التتمية المستدامة شرطا جوهريا فيها؛ وذللك لضمان متطلبات وحاجات السكان. لكنّ البُعد الاجتماعي فيها يظل هو الرّكن الرّكين، على أساس أن الهوية الثقافية والشمول الاجتماعي، يظلاّن عاملان لا غنى عنهما؛ في الأماكن التي تتمّ فيها المبادلات والنمو

بالنسبة لعملية التنمية عامّة [10].

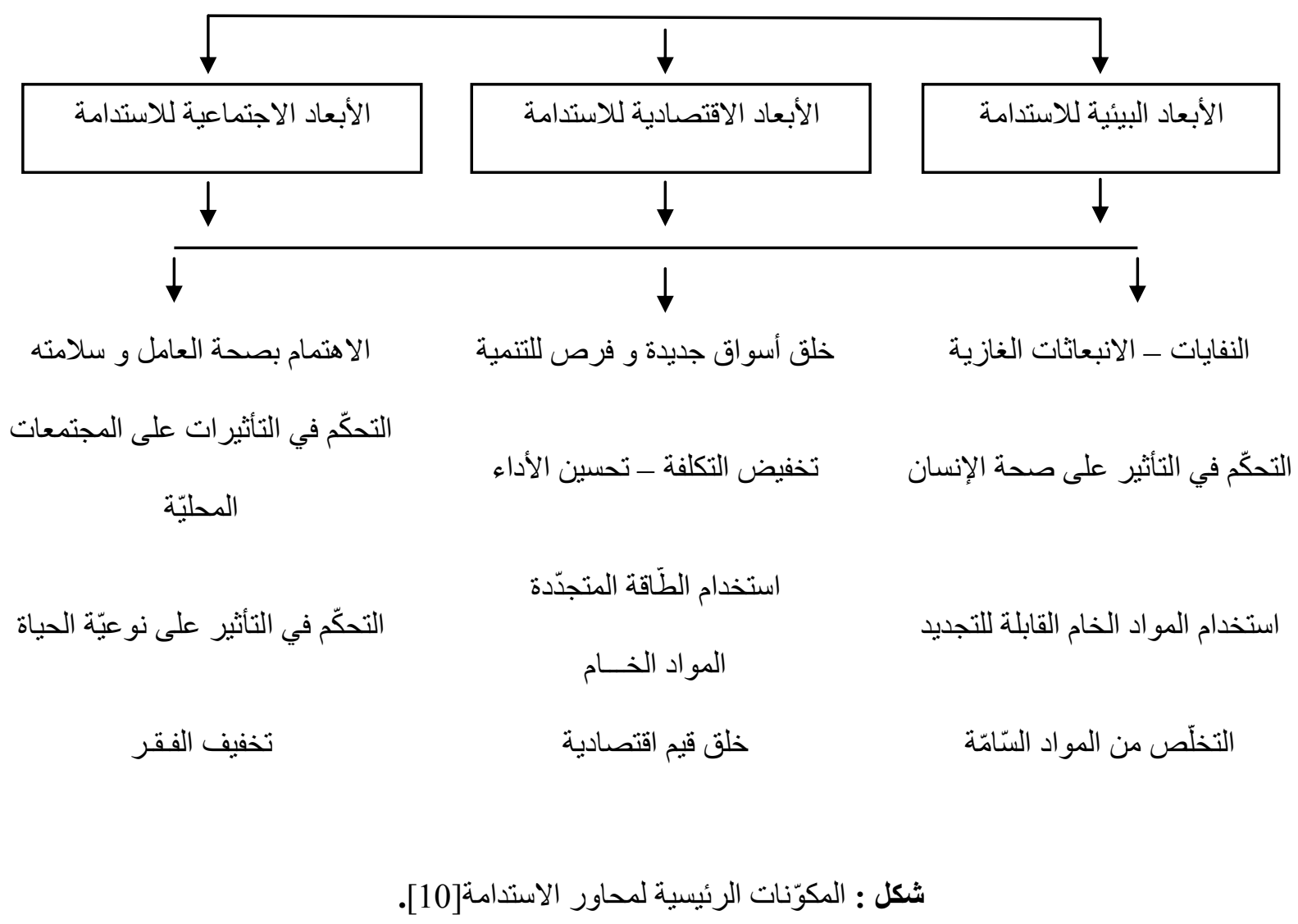

\section{2.}

يقوم مفهوم التنمية المستدامة، الذي أشرنا إليه سابقاً، على الاهتمام بين النّظم أو الأبعاد الجزئية الثلاثة؛ المتمثلة في البُعد البيئي والاقتصادي و الاجتماعي؛ فتجعل من مفهوم رأس المال بأنواعه الأربعة (الطبيعي والإنساني والمصنوع والاجتماعي) مفهوما ضروريا في مكوّنها، بينما يظهر العامل الطبيعي أو رأس المال الطبيعي كعامل مقبّ أو محدّد Constrained factor؛ إذ لا يمكن أن ينمو الاقتصاد أكثر مما تسمح به الطاقة الطبيعية، وذلك من حيث الإمداد بالموارد الطبيعية أو بلع النفايات؛ لذلك كان الثرط الأدنى لاستدامة رأس المال الضروري هو حفظ المتجدّد وغير المتجدّد منه. وزيادة على العامل الطبيعي كر أس مال مقيّ، يعترض التنمية إكراه من نوع آخر و هو التوزيع الأمثل للموارد عبر الزمن Intertemporal Allocation of Resources، وتتبني الموازنة على التكلفة والمنافع 
بين الأجيال الحاضرة والأجيال الآتية، وهي موازنة تقوم على سعر الخصم Discount Rate؛ الذي يأخذ بعين الاعتبار المصلحة العاجلة على المصلحة الآجلة، مما يَجعل من تحيّز الأجيال الحالية لمصالحها على حساب الأجيال القادمة موضع السؤال؛ خاصة وأن وُجهات النظر في تقدير المصالح والمفاسد منباينة. هذا زيادة على إمكانية ظهور بعض الآثار السالبة نتيجة الاستغلال السيئ، إلى فترة قد

$$
\text { تمتد إلى حقبة ما بعد الأجيال الحالية[11، 62]؛ وهذا ما وضع النموذج التنموي الغربي العلماني في مأزق أخلاقي عَصيْ ! }
$$

ولو أن هذا النموذج حاول إحاطة مفهوم التنمية بسياج أخلاقي و صبغة بيئية، إلا أن إصراره واستمر اره على نفس النهج المعهود

يُنْبت تمسّكه بهدف واحد هو تحقيق النمو لا غير[12]؛ أب رَهن تحقيق السعادة بالوفرة المادية، ممّا نتج عنه مفاسد بيئية واجتماعية كبيرة؛ أدّت إلى شقاءٍ ما انفكّ يتوسّع لتدفع ثمنه كل المخلوقات إورغم أنّ هذا النموذج حاول الخروج من هذه المعضلة بالتضحية بكثير من المصالح الثانوية، إلا أن المعضلة ظلت قائمة بسبب بنية النموذج المقترح ومعطياته المعرفية والقيمية؛ والتي تعكس حسب المفكر محمد الحسن بريمة إبراهيم "معطيات قلبه الصلب Hard Core" [11]. الأي قام على "عقلانية علمية" كان قصدها السيادة على الكون تنبؤاً وتحكماً وتصرفاً؛ مع الأخذ بالأخلاق العلمانية الحداثية "أخلاق السطح"، التي دأبت على قطع حبل صلتها بالأخلاق الدينية "أخلاق العُمق"، وكل القيم التي لا تثولّد من شروط التقدم العلمي والتقني[13]. وقد انجرّ عن طرح الأخلاق الدينية في هذا النموذج العلماني، أثر سلبي دفَع بالفلاسفة الأخلاقيين إلى تصوّر أطاريح أخلاقيةٌ؛؛ كان الغرض منها تصحيح الثطط التقنوعلمي. لكنّ هذه النظريات الأخلاقية، كما يرى الفيلسوف طه عبد الرّحمن، عجزت عن تقويم السيادة التي ناقثتها، فبقيت حبيسة النظريات الأخلاقية للفلاسفة القدامى، وظلّت تدور في فلك الأخلاقية التنكرية العلمانية؛ القائمة على مبدأ القطيعة مع الأخلاق الدينية الوَحْيانية.

\section{3}

تتوجّه الأخلاق البيئية إلى دراسة علاقة الإنسان الأخلاقية بمحيطه الطبيعي، وهي تنقسم إلى ثلاث أطروحات، تأخذ الأولى اسم "البيومركزية Biocentrism" أب التّمركز حول الحياة البيولوجية؛ بينما تُعرف الثانية بالأنثروبومركزية Anthropocentrisme أي التمركز حول الإنسان، في حين تجعل الأطروحة الثالثة Ecocentrism التمركز حول البيئة موضوعها. ويتلّّص مضمون الأطروحة الأولى "البيومركزية"؛ في إعطاء جميع الكائنات الحية قيمة ذاتية "داخلية" دون النظر إلى علاقتها بالعالم الخارجي؛ فالقيمة ليست مرتبطة بالعقل أو العاطفة، فهي تُركّز على توسيع وتمديد الروابط الأخلاقية إلى الكائنات الحية الأخرى، عوض حصر هذه العلاقات بين البشر فقط؛ مُركّزة على الحياة البيولوجية لإبراز أبعادها الأخلاقية. وتَعتبر الأطروحة "البيومركزية" كل الكائنات التي تعيش ضمن

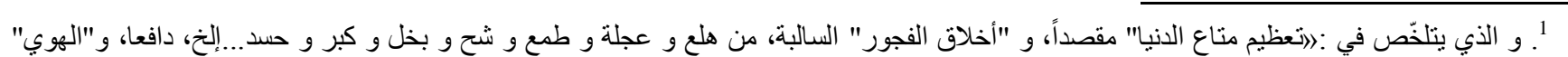

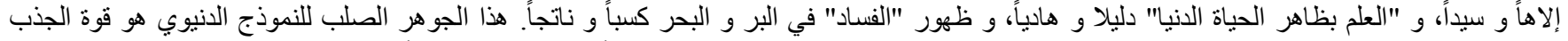

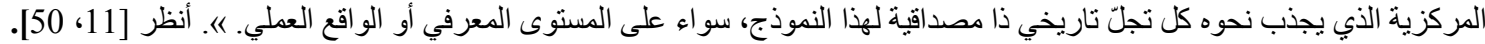

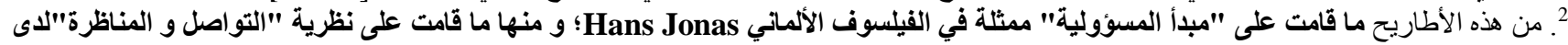


"المحيط الحيوي" The biosphere مشروعاً للحياة، ومساوية لجميع عناصر الحياة البيولوجية التي تنطوي على مضمون أخلاقي مؤدّاه احتر ام الحياة. فكل كائن حي له مقاصد أخلاقية عملية جو انية Intrinsic، لها إمكانية التّحقق من عدمها حسب الملابسات الطبيعية

أما الأطروحة "الأنثروبومركزية"، فهي ترى أن المصالح الإنسانية لا يجب النظر إلى أنها تريد التّحقق بموجب غايات مصلحية ونفعية فقط، بل إن مبعث هذه المصالح أسباب إنسانية معنوية وجمالية، وأن الإنسان لا يكون مضطر اً لإضفاء مسحة أخلاقية على مكونات الطبيعة عدا النّوع الإنساني. وقد ساهمت هذه الأطروحة في التفكر جديّا في المفارقة بين التأكيد على خصوصية الإنسان باعتباره نو عاً، وبين ما سيّبه هذا الإنسان من دمار في الطبيعة. أما الأطروحة الثالثة "الإيكومركزية"، فهي تعود لفيلسوف أخلاق الأرض عالم الغابات الأمريكي Aldo Léopold، الذي يرى بوجوب العمل على تحقيق شعار " الوحدة والاستقرار والجمال في الوسط الحي"؛ وهو مُمكن التّحقق بالنظر إلى مكونات الطبيعة ككلّ

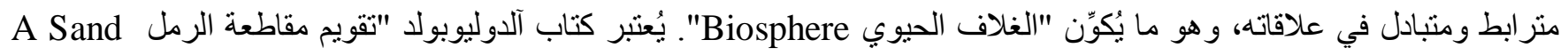
County Almanac"، الذي ظهر سنة 1949 بداية تنكيل الأخلاق البيئية من زاوية فلسفية؛ و التي تقوم على فكرة الحفاظ على المجتمع الحيوي باعتبار الإنسان كائناً مشاركا لا متحكماً وسيّداً. ويُلخّص ليوبولد فلسفة الأخلاق البيئية الجديدة من خلال انتقاده للعلاقة النفعية بين الإنسان و الطبيعة بقوله :»اإن العقبة التي تجب إز التُها من أجل إطلاق السيرورة التطوّرية للأخلاق هي ببساطة: التخلّي عن التفكير في الاستخدام اللائق للأرض وكأنه مشكلة اقتصادية حصراً، واختبار كلّ مسألة بواسطة ما هو صائب أخلاقياً وجمالياً، إضافة إلى ما هو مفيد اقتصادياً. يكون الثيء صائبا عندما يميل إلى صون تكامل واستقرار وجمال المجتمع الحيوي، و يكون خاطئًا عندما يميل إلى عكس ذلك.ب[14] وتلتقي الإيكومركزية مع الأيكولوجيا العميقة DeepEcology في فكرة ترابط منظومات الحياة على الأرض ونقدها للمركزية البشرية؛ إضافة إلى فكرة التحقُق الذاتي من خلال التّاهي مع النطاق الأيكولوجي، قصد تحسين حال الحياة على الأرض وتجنب ما من شأنه أن بَضرٌ بها. في حين تتجاوز مدرسة "التمركز حول البيئة" الأيكولوجيا العميقة بالاستناد على أسس علمية وعقلانية لبناء أخلاق بيئة جديدة. كما واجهت الايكولوجية العميقة انتقادات شبيهة بالانتقادات التي وُجّهت للأيكولوجية الدينية Ecotheology؛ وذلك باعتمادها على أفكار تتحو منحا دينيا وميتافيزيقيا حدسيا له طابع جو هري شمولي [15]Holistic]. 


\section{أ. المقاصد والاستدامة:}

مع اهتمام مفهوم التنمية بتحقيق أهداف على المستوى الاجتماعي والاقتصـادي و البيئي، معتمداً على رأس المال الطبيعي، ومنتدباً جملة من الوسائل لتحقيقها، يكون هذا المفهوم مفهوما مقاصديا معياريا بامتياز [11، 56]. الأمر الذي يجعل من التقائه مع مقاصد الثريعة مُسوَّاً؛ وهو تحقيق استدامة صلاح المُخاطَب أو المهيمَن عليه ــ أبي الإنسان- بصلاح نفسه وعقله وكرامته وماله و عرضه وصلاح موجودات العالم المُسخَّرة له، وفي هذا صلاح الأمّة و النّاس جميعاً][16] [17]. وتظهر مستويات إصلاح الإنسان في فلسفة الإسلام الأخلاقية، على مستوى الاعتقاد والتقكير، و على مستوى النفس بتَخليتها من الكدورات وشو ائب، وتحليتها وتزكيتها بالأعمال الصالحة؛ ذلك أن باطن الإنسان هو المعوّل عليه في حصول الاستعداد للتّرقي في مدارج الأعمال و الكمال، وبصلاح الاعتقاد والسلوك يكون صلاح حياة الناس وشؤونه؛؛ أي تحقيق مصالحهم المادية و المعنوية، أي كل » ما يؤثر صلاحاً أو منفعة للناس عمومية أو خصوصية.《) [18] لذلك، جاءت الشريعة لصَون الأصول الخمسة العامّة المعروفة، والتي يكون في رعايتها وحفظها تحقيق مصالح الناس، وفي فوْتها وهتكِها يكون وقوعهم في مفاسدها. وكل ملّة من الملل أو شريعة من الشرائع، ثُريد صلاح الخلق ومنافعهم، لا يمكنها بحال تفويت هذه الأصول في شعاب الحياة، و إلا جرَت مصالحهم على فساد واستحالت منافعهم إلى مضار ، و إن اختلفت نعوتها و اسماؤ ها. لذلك جعل الإمامان محمد عبده والطاهر بن عاشور مقاصد الثريعة جزءاً من برنامج الإصداح الإسلامي؛ وهذا لأن نظرية المقاصد نولي أهمية للحكمة والهحف المقصود من النص الشرعي، كما أنها نظرية تتو افق و أصول النظريات التشريعية بتحقيقها للعقلانية والأخلاق [16]. ومن هذه الرؤية يمكن للشريعة أن تأخذ بُعداً آخر وهو بُعد "الفقه الأكبر"، الذي يُضفي على الشريعة مسحة المرونة و السّعة، الأمر الذي يُخوّل لها مناقثة النازل المستجَد، فيمكنها بالتالي تحقيق مصالح الناس واستدامتها. وتظهر العلاقة بين المقاصد و التنمية المستدامة من خلال الصورة الجديدة لنظرية المقاصد، متماثية مع المستجدات المعاصرة و المفاهيم الحديثة؛ كالتي تدخل ضمن دائرة حفظ الأسرة بديلا عن حفظ النسل، أو حفظ الكر امة وحقوق الإنسان بديلا عن حفظ العرض. أو ما يمكن أن يظهر في صلة الشريعة الإسلامية مع المفاهيم العالمية المعاصرة، كحرية الاختيار والسماحة و التيسير و التكافل الاجتماعي و التنمية والمدنية، إلخ. [19].

\section{ب. أخلاقية المقاصد:}

إذا كانت الفلسفة الغربية تنظر إلى الإنسان بوصفه غاية صرفة، جاعلة من الحرية مكمن قيمته المرجعية؛ فتقع بالتالي صلته بالغيب وتُخلّده لعالم الثهادة. تمنح بالمقابل الفلسفة الإسلامية الإنسان صورة العبودية لله، من خلال مهمّة الاستئمان والاستخلاف في الأرض، و التي تسوّغ للمسؤولية الأخلاقية؛ بمظهريْ "التكليف" تحقيقا لمقصد الدّين، ومظهر " التشريف" أو "التكريم" الذي هو حال 
اصطباغ الإنسان بالأمن و الهداية.فالكر امة الإنسانية هنا تبدأ من صفة التكريم، وتتأصل بصيغة التكليف، و عليه نؤَّف الأخلاق عند هذا الشخص اعتبار وامتثالا [20]. فالرؤية الأخلاقية الإسلامية تُنكّر الإنسان بمقاصد الحق من الخَق، بهدف إصابة غاية خُلقية مفادُها تزكيّة النّفس بالدين؛ الذي هو 》 التخلّق بمكارم الأخلاق《 كما قال الإمام الشاطبي [18]. و لا يُفهم من "مكارم الأخلاق"، كما شاع في المصادر الأصولية، أنها من قبيل الأمور التحسينية التي لا يكون في تركها حرج أو مشقّة، بل هي من الضروريات التي لا تقوم حياة الإنسان إلا بها؛ وفي الاستغناء عنها فساد لا مناص منه، كما لا يُتصوَّر أن يكون تنزيل الشريعة لغرض تتميم أخلاق تحسينية فقط ![21] فالأخلاق على رأى الفيلسوف طه عبد الرحمن هي ما يُحقّق ماهية الإنسان؛ لأن خليقة الإنسان لا تَتحقّق إلا بالخَلق والخُلق، و إذا كانت ماهية الإنسان تقتضي الخُلقية، فلا إنسانية بغير أخلاقية. ففي مُصساحبة الإنسان للأخلاقية تحقيق لعالمية الإنسان بالأخلاق، أو تعميم للأخلاق الإنسانية الطارحة للاسترقاق العلمي[22]. وتظهر منزلة الأخلاق من الشريعة بمنزلة القلب من الجسد، فلا غَروَ أن نجد تقسيم المقاصد الشرعية، على اعتبار درجة تحقيق الشرط الأخلاقي فيها. وتعمل كلية الدّين عمل الرابط للضّروريات الخمس، وهي مناط حفظها؛ لأن الدين ـ الذي هو حُسن الخُلقـ هو المقصد الأخلاقي الأسمى في النظرة المقاصدية الثاملة؛ التي لا تَخرج عن كونها جلب مصلحة أو دزء مفسدة3. ومفهوم المصلحة ليس " اسم ذات متحيزة " بل هو اسم معنى مُجرّد بمعنى "الصّّلاح"؛ يُشنير إلى قيمة خُلقية تُسوِّغ وصف مقاصد الشريعة بـ "علم الصلاح" الإنساني في العاجل والآجل، أبي في الدّنيا والآخرة[23] [18،8]. وعليه، يكون مبحث المقاصد مبحثا يجعل من مقاصد الشريعة علماً أخلاقيا؛ عديلا لعلم القيم أو "الأكسولوجيا" ؛ فينظر في الأفعال التي يَّور حولها الحًكم الثرعي اعتبارًا أو إلغاءً؛ من جهة صلاحها الذي يَنضوي ضرورة على قيمة أخلاقية؛ هي في النهاية مقصد الثارع الحكيم. لذلك يَتقرى الأصولي في الأحكام القيم الكبرى أو المقاصد التي تَنبني علبها نكاليف الدين، أي القيم المستدامة على شرط الاستقر ار والصدق، مساهمة منه بالجواب على السؤال القيمي الحائر في بُعده الكوني و الوجودي[24].

\section{ت. مدلول التنمية بروية مقاصدية:}

كان المأزق الأخلاقي في النموذج التنموي الغربي، مسوّغا لضرورة تصوّر نموذج بديل عنه؛ وهذا بالنظر إلى الإخفاقات التنموية التي خلّفها و النُّذر البيئية التي ثُلوّح عن كارثنة وشيكة ! وعليه، ذَعت الضرورة إلى انتداب نموذج آخر يختلف جوهريا عن مكوّنات قلب النموذج الغربي الصلب؛ و الذي لا يمكن أن يكون إلا النّموذج التنموي الإسلامي التوحيدي.

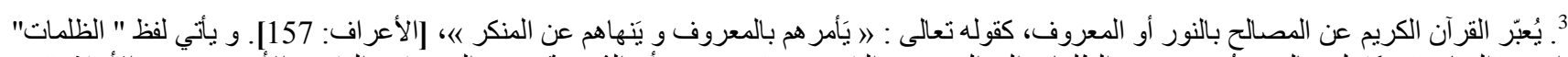

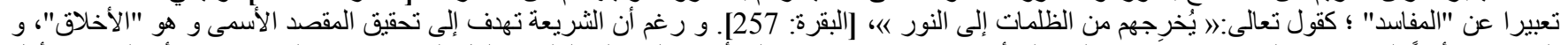

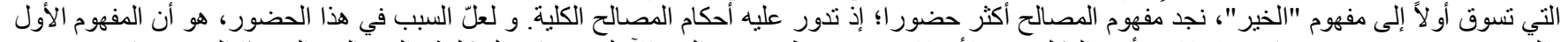

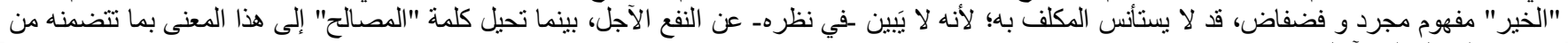


يتساوق مفهوم التتمية المستدامة مع فلسفة الإسلام الكونية، التي تقوم على فكرة الاستخلاف و عمارة الأرض؛ ومفهوم الاستخلاف و عمارة الأرض مفهومان قر آنيان، يَجدان بعض مكوّناتهما في ما يقابلهما من المفهوم الغربي لمصطلح التنمية المستدامة، لكن المفهوم القر آني لهذين المفهومين يتعدّاه ويتجاوزه بخصوصية أو شرط المسؤولية الأخلاقية الثناوية فيه؛ والتي تقضي بضمان استمرار صلاح الأرض بصلاح المستخلَفين فيها. ولا يمكن لهذه العمارة و هذا الصلاح، أن يتحققا إلا على وفق النموذج التوحيدي الإيماني والمنوال المقاصدي، الذي يُمنّل علم الأخلاق الإسلامي ومنظومته القيمية، والذي أشرنا إليه أعلاه بـ "علم الصلاح". ومُبرّر ذلك التقاطع تلاقي فضاءات التنمية المستدامة الثلاثة (الاجتماعي والبيئي والاقتصادي)، والمعبِّرة عن أصول الظاهرة الاجتماعية والمعادلة لخصائص القرآن الكريم المعرفية من جهة ؛ مع أصول الثريعة وكلياتها في بُعدها العملي و الأخلاقي و التوحيدي من جهة أخرى [11،11]. ورغم اشتر الك النموذج التوحيدي مع النموذج العلماني الغربي في الهدف التتموي، المتمثّل في تحقيق الحياة السعيدة والرّفاه، أو الحياة الطيبة بالتعبير القرآني، تتجلّى خصوصيّة النموذج التنموي التوحيدي في صفة "الإيمان"، الذي هو مؤشر المتغيرات الكونية (النفس والعالم و المال و البنون)، و الذي يتجلّى عمليا في زينة الحياة الدنيا من خلال قيمتين أخلاقيتين عمليتين هما "الثكر والصبر"، و اللتان تنوبان عن الإيمان في عالم الواقع والفعل اليومي؛ بالعمل الصالح الذي يُراعي فيه المكلَف قصد الثشارع الحكيم. كما يجعل النموذج التوحيدي من الإيمان والعمل الصالح وسيلتين في صميم العملية التنموية، لتحقيق الطمأنينة ووُسع العيش والبركة في حياة المجتمع المؤمن؛ مقابل الحياة الضنكة التي يكون سببها الكفر بأوسع معانيه، ومُترجَماً بالعمل السيئ بكل أوجهه [11، 72]. فشرط

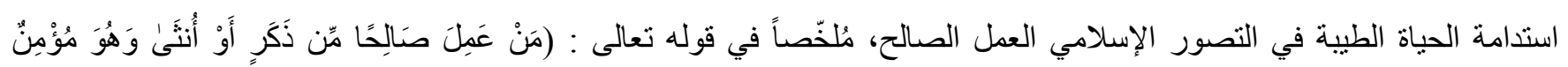

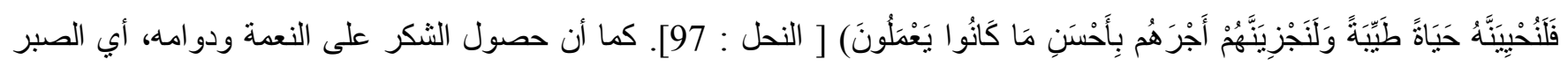

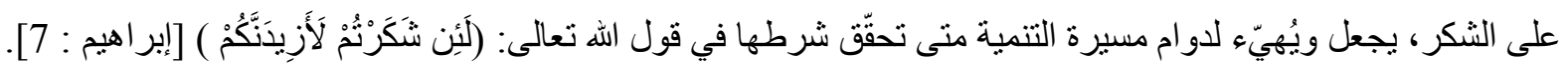
فزيادة على أخلاق الثكر التي تتجلّى من اعتمال الإيمان في القلب وتصديقه بالعمل الصالح، يلتزم المؤمن مسيرة الاستدامة الرّاشدة، التي لا تتعلّق بكفاءة العقل الأداتي ومصداقية الوسائل لديه وحسب، بقدر ما يكون تُحقيقها لمقاصد الشارع ومُر اده في العاجل و الآجل؛ قصد درء المفاسد الذي يُحقق شرط حفظ المصالح وجودا، أو جلب المصالح بمعنى استدامة ما تمّ حفظه؛ ذلك أنّ ما لا يُمكن حفظه أو لاً لا يُكن استدامته، على اعتبار أن الحِفظ شرط أوّلي في تحقيق الاستدامة. و لأن أصول الظاهرة الاجتماعية مساوية لمكوّنات المقاصد الكلية الثرعية، فهي تأخذ ـ في مصطلح الاستدامة ـ حُكم المتغيرات القابلة للزيادة أو النقصان في الكّّ والكيف، فكان من Temporarily Non- الضروري إذاً حفظها أو استدامتها بتنميتها؛ تلافيا لنقصانها أو تراجعها وضمور ها عبر الزمن على الأقل declining و إلا استحالت تتميتهال11، 72]. 
و لأنّ عامل الزّمن في البحث التتموي مهم للغاية، فهو يُولي نتائج الأعمال ومقاصدها في المدى البعيد أهمية كبيرة؛ ومنه يُمكن ملاحظة تميّز النموذج التنموي الإيماني، باستحضاره من جهة، للبُعد الغيبي باعتبار الوحي مصدرا نوعيا و حاسما؛ يمكنه أن يُغني المكلّف عن الوقوع في الفساد أو أخلاق الفجور الهادمة[25]. واستصحابه من جهة أخرى، لطبيعة الفعل الإلهي متجاوزاً لمفهوم الزمن العاجل؛ قد تظهر نتائجه في الآجل بحسب طبيعة الفعل البشري؛ شُكوراً أو كُفوراً [11،96]. كما تظهر عناية المؤمن بالزمن، عند عملية المداولة في اختيار الفعل والترجيح في اتخاذ القرار، جاعلا نصب عينيه أوّلا قيمة عمله في الدارين؛على نقيض اهتمام ونتائج النموذج العلماني الذي يولي اهتماما بالمصالح العاجلة فقط. هذا زيادة على أن امتداد التعاون في الزمن، حسب النموذج الإيماني، أذْعَى وأجْلب للمصالح العامة الآجلة على المصالح الخاصة العاجلة؛لسبب قيام التعاون على التنافس في البِر والتقوى[11، 82-83]. خاتمة : يتبيّن لنا ممّا تقدّم، أن مفهوم التنمية المستدامة مفهوم غبر قادر، فهولا يلبثثُ يتطّور بتطوّر الفكر الاقتصادي وفلسفة العولمة التي أفرزته. وقد ظلّ يتشكّل باحثا عن صورة تتجاوز مفهوم النمو إلى التنمية؛ وذلك بمحاولة إيجاد موازنة وتكافؤ بين الجانب المادي و المعنوي لحاضر الإنسان ومستقبله. لكنّ الفكر الاقتصادي أظهرعجزا في تتاول المفهوم بكلّ شموليته وحيثياته الإنسانية، خاصّة في بُعده المعنوي و الأخلاقي الرّوحي؛ والذي يُعدّ شرطاً مُهمّا حسب تصوّر النموذج الإسلامي للتنمية. و لأن مفهوم الاستدامة مفهوم معياري يَنظر في استر اتجيات التنمية ومآلاتها، يُمكن لعلم المقاصد أن يكون صيغة جديدة أو نموذجا مناسبا وبديلا عن منوال النموذج الغربي؛ من جهة اعتبار مشاركته في المحافظة على الضروريات و المصالح المُنّقق عليها إنسانياً حسب مبدأ "عدم الإضرار" أو لاً، و من جهة تجاوزه له بمضامين أخلاقية دينية وأبعاد روحية ثانياً. هذا، زيادة على أن المقاصد المُر اد إصابتها في النموذج التنموي الإسلامي، هي مقاصد يَدلّ عليها ضابط المصلحة و الصلاح في الدنياو الآخرة، مقابل المقاصد التي ينوقف تحقُقها في النموذج الغربي، على مدى إصابتها من زينة الحياة الدنبا وفق ضابط الهوى. لذلك دَعت الحاجة لتغيير تصوّر النّموذج الغربي عن التنمية؛ والذي تأسّس على تصوّره المُفتقَر عن الكون والإنسان، من أجل استئناف موقف اجتهادي لتقديم فلسفة الإسلام؛ من خلال بحث عميق وتناول دقيق، لعلاقة الإنسان بالكون و الغيب وفق طرح أكثر إثراءً و إرواءً على صعيد التجربة الأخلاقية و الرّوحية؛ قصد المساهمة في تصويب التجربة الإنسانية، معرفيا وقيميا، مسترشدين في ذلك بمناهج العقل و إلهامها ومهتدين بنور الوَحي الإلهي. 


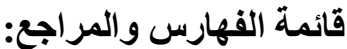

[1] العيسوي، إبر اهيم. التنمية في عالم متغير دراسة في مفهوم التنمية و مؤشر اتها.القاهرة : دار الثروق، 2000، ص، 18.

[2] United Nations World Commission on Environment and Development (UNWCED). Our Common Future. Oxford UniversityPress, 1987.

[3] بن منصور، عبد الله الجوانب الأخلاقية في التنمية المستدامة، مجلة271، Les Cahiers du MECAS،2008 ، ع 4، 361-372، ص

[4] بلقاسم، سهام. دور الاستتمار السياحي في تحقيق التنمية المستدامة. رسالة ماستر.الجزائر: جامعة أكلي محند أولحاجالبويرة، 2017، ص، 25.

] Repetto, Robert. Word Enough and time. Yale University Press. New Haven, CT. 1986, pp. 15-16.5[

[6] Perreux. François, L'économie du XXe siècle. Grenoble : PUG, 1991. [7] مهران. هشام، التوظيف الأمنل للمحددات الطبيعية لتخطيط بيئة عمرانية منوازنة. ندوة الإبداع و التميّز في النهضة العمر انية،

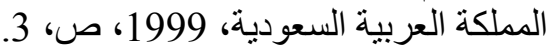

[8] Sen. Amartya, Un nouveau modèle économique. Développement, justice, liberté, Paris : Odile Jacob, 2000, p.36.

[9] www. Sustainability.com. What is Sustainable Developpement.10/2005. [10] هجيرة، سعودي. التتمية المستدامة من خلال المبادئ المبادئ العمر انية للمدن العتيقة، دراسة حالة مدينة بوسعادة، رسالة ماجستير ،الجز ائر:

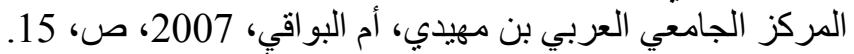

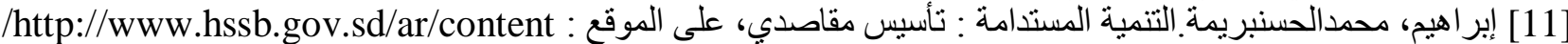

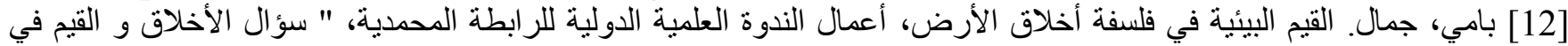

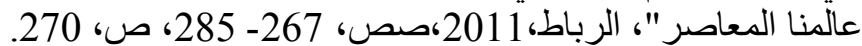

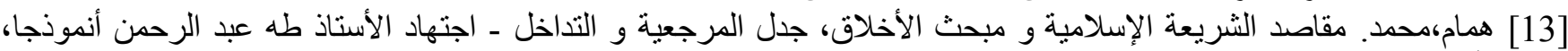

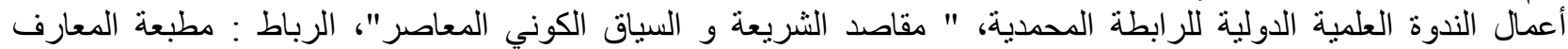

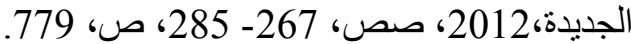

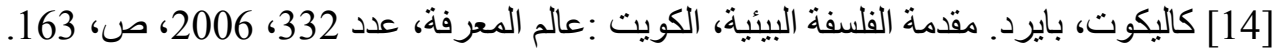

BLAIS, François ; FILION, Marcel. De l'éthique environnementale à l'écologie politique. [15]

Apories et limites de l'éthique environnementale. Philosophiques, 2001, vol. 28, n², p. 255-280. [16] هيتوت، عبد الله، معالم النظرية المقاصدية، أعمال الندوة العلمية الدولية للر ابطة المحمدية، " مقاصد الثريعة و السياق الكوني

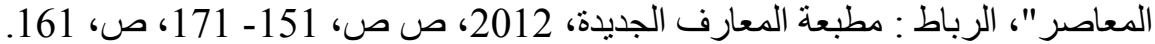
[17] ابن عاثشور، الطاهر. مقاصد الثريعة، تونس: دار سحنون للنشر و التوزيع ؛ دار السلام للطباعة و النشر و التوزيع، 2009،ص،

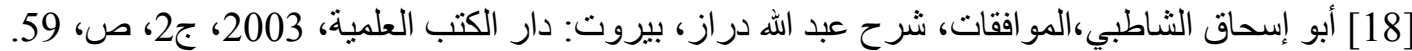

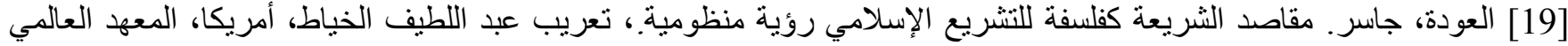

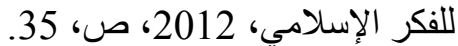

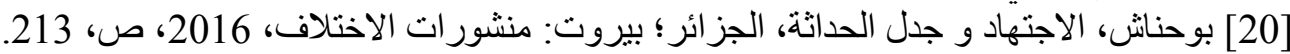

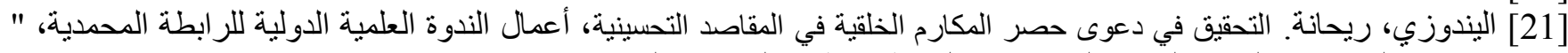

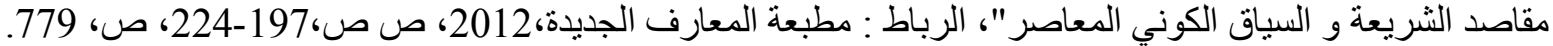
[22] عبد الرحمن، طه. سؤال الأخلاق، الدار البيضاء، المغرب ؛ بيروت، لبنان : المركز الثقافي العربي، ط2، 2000؛ ص ص، ص، 14؛ .65 54

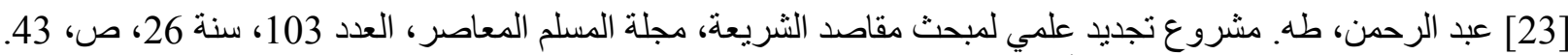

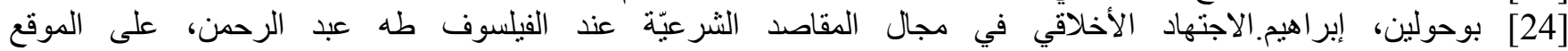
:Www.mominoun.com/articles :

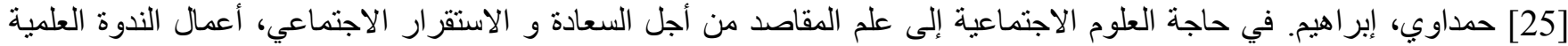

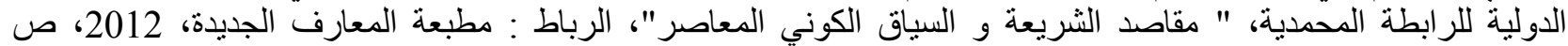
ص، 461-474-47، صن، 469. 